\title{
Polymorphisms at the $F 12$ and KLKB1 loci have significant trait association with activation of the renin-angiotensin system
}

Nilima Biswas ${ }^{1 *}$, Adam X. Maihofer ${ }^{2}$, Saiful Anam Mir ${ }^{1}$, Fangwen Rao ${ }^{1}$, Kuixing Zhang ${ }^{1}$, Srikrishna Khandrika ${ }^{1}$, Manjula Mahata', Ryan S. Friese', C. Makena Hightower', Sushil K. Mahata 1,3, Dewleen G. Baker 2,3,

Caroline M. Nievergelt ${ }^{2,3^{*}}$, Sucheta M. Vaingankar ${ }^{1 *}$ and Daniel T. O'Connor ${ }^{1,4}$

\begin{abstract}
Background: Plasma coagulation Factor XIla (Hageman factor; encoded by F12) and kallikrein (KAL or Fletcher factor; encoded by $K L K B 1$ ) are proteases of the kallikerin-kinin system involved in converting the inactive circulating prorenin to renin. Renin is a key enzyme in the formation of angiotensin II, which regulates blood pressure, fluid and electrolyte balance and is a biomarker for cardiovascular, metabolic and renal function. The renin-angiotensin system is implicated in extinction learning in posttraumatic stress disorder.

Methods \& Results: Active plasma renin was measured from two independent cohorts- civilian twins and siblings, as well as U.S. Marines, for a total of 1,180 subjects. Genotyping these subjects revealed that the carriers of the minor alleles at the two loci- F12 and KLKB1 had a significant association with reduced levels of active plasma renin. Meta-analyses confirmed the association across cohorts. In vitro studies verified digestion of human recombinant pro-renin by kallikrein (KAL) to generate active renin. Subsequently, the active renin was able to digest the synthetic substrate angiotensinogen to angiotensin-I. Examination of mouse juxtaglomerular cell line and mouse kidney sections showed co-localization of KAL with renin. Expression of either REN or KLKB1 was regulated in cell line and rodent models of hypertension in response to oxidative stress, interleukin or arterial blood pressure changes.

Conclusions: The functional variants of KLKB1 (rs3733402) and F12 (rs1801020) disrupted the cascade of enzymatic events, resulting in diminished formation of active renin. Using genetic, cellular and molecular approaches we found that conversion of zymogen prorenin to renin was influenced by these polymorphisms. The study suggests that the variant version of protease factor Xlla due to the amino acid substitution had reduced ability to activate prekallikrein to KAL. As a result KAL has reduced efficacy in converting prorenin to renin and this step of the pathway leading to activation of renin affords a potential therapeutic target.
\end{abstract}

Keywords: FXIla (active protease encoded by gene F12 or Hageman factor), Kallikrein/KAL (active protease encoded for by gene KLKB1 or Fletcher factor), rs3733402, rs1801020, PTSD (posttraumatic stress disorder), Hypertension

\footnotetext{
*Correspondence: nbiswas33@gmail.com; cnievergelt@ucsd.edu;

svaingankar@ucsd.edu

1Departments of Medicine, University of California at San Diego, 9500 Gilman

Drive, La Jolla 92093-0838, USA

${ }^{2}$ Veteran Affairs (VA) San Diego Health Care System and VA Center of

Excellence for Stress and Mental Health, 3350 La Jolla Village Drive, San

Diego 92161, USA

Full list of author information is available at the end of the article
} 


\section{Background}

Hypertension is a global public health issue and contributes to the burden of heart disease, stroke, kidney failure and premature mortality (13\% of total deaths worldwide)[1]. The kidney serves as a major organ for maintaining normal blood pressure (BP) and the local renal renin angiotensin system (RAS) pathway acts as the master regulator of renal function during hypertension [2-4]. The renin-angiotensin-aldosterone system (RAAS) is a signaling pathway responsible for regulating the body's blood pressure [5-8]. Stimulated by low BP the kidney releases renin, this triggers a signal transduction pathway generating eventually angiotensin II that causes vasoconstriction, leading to increase in BP. Several cardiovascular therapies for high BP, target the RAAS system and these therapies are now being explored for their efficacy in treating PTSD $[9,10]$.

The juxtaglomerular (JG) cells in the kidney express renin a member of the aspartyl protease family. It is the limiting enzyme in RAS pathway that converts angiotensinogen to angiotensin I (Ang I) [11]. Renin production is tightly regulated at the transcriptional level and the active renin is released into the circulation through regulated exocytosis [11, 12]. About $80 \%$ of the renin present in plasma is in an enzymatically inactive form called pro-renin. Kidney processes inactive pro-renin to renin and is the major source of circulating active renin in humans. The plasma renin concentration contributes significantly to cardiovascular and renal diseases like hypertension, coronary heart disease, and chronic kidney disease [13]. Thus the conversion of pro-renin to renin is a potential regulatory site for therapeutic intervention.

We studied the effect of the KLKB1 (located on chromosome 4) missense variant rs3733402 (Asn124Ser) on circulating levels of active renin and observed that homozygous carriers of the minor allele (Ser/Ser) displayed lower levels of active renin [14]. In vitro proteolysis and cell biology indicated that pro-renin was a substrate for plasma kallikrein (KAL). The KAL-activated renin in turn, was able to cleave substrate angiotensinogen to angiotensin 1 the precursor for vasoconstrictor angiotensin II. Situated on chromosome 5, the coagulation factor F12 5'-UTR variant rs1801020 also showed significant association with plasma levels of active renin. The F12 locus encodes for the FXIIa protease responsible for converting pre-kallikrein to KAL. The possible implication of the intrinsic coagulation system and the fibrinolytic system in renin activation has been discussed. In both the independent cohorts a strong association was observed between levels of active renin and occurrence of the minor alleles.

\section{Methods}

\section{Twin and sibling subjects}

Twin and sibling participants (TSP) for the human study were recruited from southern California by access to a population birth record-based twin registry [15], as well as by newspaper advertisement [16]. The University of California San Diego, Institutional Review Board provided approval for the study and each subject or the parent of the minor subjects, gave written informed consent. A subset of 381 individuals of the TSP population was randomly selected and included 60 dizygotic (DZ) and 160 monozygotic (MZ) twin pairs. Zygosity of twins was confirmed genetically by use of microsatellite and single nucleotide polymorphism (SNP) markers [16]. Initially ethnicity was established by self-identification, including information on geographic origin of both parents and all four grandparents, and only individuals of Caucasian or Hispanic ancestry/ethnicity are included here. The age of the subjects ranged from 14 to 78 years, with a median of 39 . Phenotyping (biochemical and physiological) was conducted as previously described [16]. All of the 381 TSP subjects with both genotypes and phenotypes were included in the analyses (see below).

\section{Molecular genetics, genomic DNA and genotyping}

Genomic DNA was extracted from leukocytes in EDTAanticoagulated blood after proteinase- $\mathrm{K}$ digestion of proteins, by adsorption/elution from Qiagen columns, as previously described [16], and genotyped for 592,312 SNPs using the Illumina 610-Quad genotyping array and passed final quality control (QC: see below). For each MZ twin pairs, only one individual underwent GWAS, and the genotype information was used for both members of MZ twins. During analysis, family structure was accounted for in MERLIN (see below).

\section{Biochemical assay of active renin in human plasma}

EDTA-anticoagulated plasma samples were collected from seated subjects, and stored frozen at $-70{ }^{\circ} \mathrm{C}$ until assayed. Circulating active renin was quantified at room temperature for 3 hours with a 2-site IRMA [17] wherein the mouse monoclonal anti human renin antibody was specific for a renin epitope formed after excision of active renin from pro-renin (DSL, Webster, TX; DSL-25100); the active renin assay sensitivity was $\sim 0.48 \mathrm{pg} / \mathrm{ml}$, with intraassay coefficients of variation from 1.4-4.3\%, and interassay coefficients of $1.9-3.0 \%$.

\section{Genetic association analyses}

To test SNP on phenotype effects with explicit accounting for family structure for the TSP cohort, MERLIN v1.1.2 (http://www.sph.umich.edu/csg/abecasis/merlin/) was used. As an additional QC step, unlikely genotypes based on expected inheritance patterns were removed using Merlin's Pedwipe procedure. A maximum likelihood estimation test of a variance components model was used, incorporating a variance-covariance matrix that allows for family relatedness, including twin status, 
to be modeled and appropriately controlled for in the association test. In addition, age, gender, and the first MDS component were included as covariates. A standard criterion of $\mathrm{p}<5 \times 10^{-8}$ across the genome was used to indicate significance of single SNPs on traits. The "Manhattan" plots visualized results across the genome, as well as local "SNAP" (SNP Annotation and Proxy Search) plots [18] <http://www.broadinstitute.org/mpg/ snap/ldplot.php>.

\section{Replication Marine Resiliency Study (MRS)}

We also measured active plasma renin (by ELISA) in samples from 799 healthy unrelated male Marines from the Marine Resiliency Study (MRS) with available genotypes [19]. The method for genotyping of MRS subjects has been detailed earlier [20, 21]. In brief, genotyping was carried out using the HumanOmniExpressExome (HOEE) array with 951,117 loci from Illumina (http:// www.illumina.com/), resulting in a high initial locus success rate and overall data quality. Additional data cleaning was performed in PLINK v1.07 [22], using standard procedures. All subjects included here were active duty male and of European ancestry [23]. All subjects provided written consent for the genetic study. Association of plasma renin activity with genotypes were performed using a linear regression in PLINK (v.1.07) using age and 3 principal components ( $\mathrm{PC}$ 's) to correct for population stratification as covariates. We used the Genetic Power Calculator from Purcell et al. to estimate power [24]. Based on an effect size estimate of $1 \%$ of variance explained by a candidate variant, we estimate that we had $83 \%$ power to detect an effect of SNP on renin levels at an alpha level of 0.05 , given the number of samples available in the MRS. Furthermore we estimate that we would have $>94 \%$ power to detect an effect of this size in a meta-analysis of the MRS and TSP.

\section{Meta-analyses}

Results from the TSP and MRS data were combined in an inverse variance and weighted fixed-effect metaanalysis was carried out using METAL [25].

\section{Protein chemistry and enzymology}

\section{Digestion of recombinant human pro-renin by human KAL}

Recombinant human pro-renin $(5 \mu \mathrm{M})$ (Cayman Chemical, catalog number 10007599) was digested with protease human KAL (kallikrein, human plasma, Calbiochem, EMD Millipore, catalog number 420307, specific activity $15 \mathrm{U} / \mathrm{mg}$ protein) $(1 \mu \mathrm{M})$ at $37^{\circ} \mathrm{C}$ for $15 \mathrm{~min}$ in $12 \mu \mathrm{l}$ of reaction volume with assay buffer $(50 \mathrm{mM}$ Tris, $\mathrm{pH} 7.5$, $\mathrm{NaCl} 250 \mathrm{mM})$. The reaction was terminated by adding aprotinin $(2 \mu \mathrm{M})$, purified by ZipTip (small C-18 column) and then analyzed by MALDI-TOF. For SDS-PAGE, prorenin was incubated in absence or presence of KAL as mentioned above for 2 hours, and analyzed on $10 \%$ or $4-12 \%$ (gradient) NuPAGE gels.

\section{Digestion of renin substrate angiotensinogen (AGT) with KAL-activated renin}

Human pro-renin $(5 \mu \mathrm{M})$ was digested with KAL $(1 \mu \mathrm{M})$ in $50 \mathrm{mM}$ Tris, $\mathrm{pH} 7.5$ and $\mathrm{NaCl} 250 \mathrm{mM}$ in a volume of $12 \mu \mathrm{l}$ for $15 \mathrm{~min}$ at $37^{\circ} \mathrm{C}$, as mentioned above in the first step. In the second step, $12 \mu \mathrm{l}$ of sodium acetate buffer, pH 5.5 containing angiotensinogen synthetic tetradecapeptide (14 amino acids; DRVYIHPFHL $\downarrow$ VIHN) (Phoenix Pharmaceuticals, Inc.) was added (in final concentrations of sodium acetate $0.2 \mathrm{M}$ and tetradecapeptide $10 \mu \mathrm{M}$ ), and further incubated for another $15 \mathrm{~min}$ at $37^{\circ} \mathrm{C}$. The reaction digests were then purified through ZipTip adsorption/elution, and were analyzed by MALDI-TOF.

\section{MALDI-TOF analysis}

MALDI-TOF analyses were performed as described before using a PE Biosystems Voyager DeSTR MALDI-TOF mass spectrometer (Applied Biosystems, Foster City, CA) [26]. Resulting peptide masses were analyzed in the ProteinProspector Program (<http://prospector.ucsf.edu $>$ ) to identify the possible fragments of the respective proteins.

\section{Identification of active renin and pro-renin protein bands in} $K A L$ digests, analysis by LC-MS/MS sequencing

Gel slices were cut, processed for in-gel trypsin digestion and the extracted peptides were analyzed by reverse-phase liquid chromatography (LC) in combination with tandem mass spectrometry using electrospray ionization with a QSTAR-Elite hybrid mass spectrometer (AB/MDS Sciex) as described before [27]. Peptide identifications were made using the Paragon algorithm executed in Protein Pilot 2.0 (Life Technologies).

\section{Amino acid sequence analysis by TOF/TOF}

Tandem mass analysis (MS/MS) for sequencing was performed on a 4800 MALDI-TOF-TOF mass spectrometer (Applied Biosystems) as described before [26].

\section{Mouse juxtaglomerular cell culture}

Mouse kidney juxtaglomerular cells As4.1 (ATCC ${ }^{\circ} \mathrm{CRL}$ $2193^{\text {mit }}$ ) were grown in DMEM high-glucose (GIBCO) with $10 \%$ FBS and Penicillin/streptomycin/glutamine media at $37^{\circ} \mathrm{C}$ with $5 \% \mathrm{CO}_{2}$.

\section{Co-localization of Renin and KAL by immunofluorescence} Mouse CRL-2193 (As4.1) juxtaglomerular cells

Cells were grown on cover slips, washed with PBS and were fixed with $2.5 \%$ paraformaldehyde in PBS for $20 \mathrm{~min}$ at room temperature. Cells were then permeabilized with $0.5 \%$ Triton in PBS for $10 \mathrm{~min}$ at room 
temperature. Cells were blocked using $5 \%$ BSA in PBS for $30 \mathrm{~min}$ followed by primary antibody incubation [rabbit anti KAL (1:100, Bioss) and goat anti renin (1:100, Santa Cruz Biotechnology)] in $2 \%$ BSA for $2 \mathrm{hr}$ at room temperature. Coverslips were washed 3 times 5 min each and then incubated with secondary antibody Alexa Fluor $488 \mathrm{~nm}$ (green) coupled to donkey anti rabbit (1:250, Invitrogen) and Alexa Fluor $594 \mathrm{~nm}$ (red) donkey anti goat (1:350, Invitrogen) along with Hoechst 33342 (nuclear stain; $1 \mu \mathrm{g} / \mathrm{mL}$ ) in $1 \%$ BSA for $1 \mathrm{hr}$ at room temperature. Coverslips were washed and mounted on glass slide using Slowfade-antifade (Molecular Probes). Images were acquired on a Delta Vision deconvolution microscope and SoftWorx software (Applied Precision, Issaquah, WA), using 60x objective as described previously [28].

\section{Mouse kidney immunohistochemistry}

Formaldehyde-fixed paraffin-embedded kidney tissue sections were cleared of paraffin and hydrated through graded alcohol and boiled in $100{ }^{\circ} \mathrm{C}$ for $20-30 \mathrm{~min}$ for antigen retrieval [29]. After permeabilization and blocking, sections were incubated overnight at $4{ }^{\circ} \mathrm{C}$ with primary antibodies to renin and $\mathrm{KAL}$, followed by incubation with Alexa Fluor secondary antibodies as described above. Images were captured on a Delta Vision deconvolution microscope using $20 \mathrm{x}$ objective.

\section{REN and KLKB1 mRNA expression in organs and cells}

Transcriptomes of mouse adrenal gland from mouse strains blood pressure high $(\mathrm{BPH})$ and blood pressure low (BPL) (each in triplicate) [30]; rat adrenal gland (SHR and WKY strains, each in triplicate) [31] and mouse As4.1 juxtaglomerular cells (in duplicate) [32] were profiled by microarray analysis as previously described, and data are available at NCBI GEO. Data were globally normalized to median expression, and then analyzed statistically.

\section{Statistical analyses}

The results were expressed as mean \pm one SEM. Multiple comparisons were made using one-way ANOVA followed by Bonferroni post hoc tests, or by two-way ANOVA using Kaleidagraph (Synergy Software, Reading, PA). Statistical significance was concluded at $p<0.05$.

\section{Results}

Meta-analysis of genetic association for polymorphisms at the $F 12$ and $K L K B 1$ loci and active renin concentration in plasma

The best-characterized functional polymorphism at the KLKB1 locus rs3733402 results in loss-of-function amino acid substitution Asn124Ser [33]. This substitution in the apple 2 domain impairs binding and digestion of the classical substrate HMWK (high molecular weight kininogen) [14]. At the F12 locus, the rs 1801020 polymorphism is in the 5'-UTR (C46T) creates a new upstream translational start codon, thereby attenuating formation of the authentic F12 protease [34].

Since these proteases are part of the kallikrein-kinin system and interact with each other at the molecular level, we looked at genetic association of the polymorphisms described above with levels of active renin in plasma. The effect of the human polymorphisms rs3733402 in the KLKB1 locus and the rs1801020 in F12 locus were very significant on the active renin levels in plasma of both the TSP and MRS populations (Table 1, Fig. 1). In both cases, minor alleles were associated with low levels of active renin in the plasma (Fig. 1). Meta-analysis combining the TSP and one independent population (MRS) for a total of $n=1,180$ subjects, indicated allelic effects consistent in magnitude (beta, or effect size per allele) and direction (sign on slope) across populations. The overall slope of the meta-analysis regression for rs3733402 and $\mathrm{rs} 1801020$ was beta $=0.055$ and 0.057 , with $\mathrm{SE}=0.014\left(\mathrm{p}=6.83 \times 10^{-5}\right)$ and $=0.016$ $(p=0.0003)$ respectively (Table 1$)$.

Table 1 Meta-analysis of the effect of KLKB1 and F12 genetic polymorphisms on generation of active renin in human plasma

\begin{tabular}{|c|c|c|c|c|c|c|c|c|c|c|c|c|c|c|}
\hline \multicolumn{15}{|l|}{ KLKB1 (rs3733402) } \\
\hline Cohort & $\mathrm{A} 1$ & $\mathrm{~A} 2$ & N & BETA & SE & P & MAF & HetISq & HetP & G/G Freq & G/A Freq & A/A Freq & HWE chi-square & HWE-p \\
\hline TSP & G & A & 381 & -0.071 & 0.025 & 0.005 & 0.5 & & & $20.42 \%$ & $49.17 \%$ & $30.42 \%$ & 0.399 & 0.712 \\
\hline MRS & G & A & 799 & -0.048 & 0.016 & 0.0037 & 0.47 & & & $20.40 \%$ & $52.82 \%$ & $26.78 \%$ & 2.67 & 0.102 \\
\hline Meta-analysis & G & A & 1180 & -0.055 & 0.014 & $7.22 \mathrm{E}-05$ & 0.48 & 0 & 0.507 & & & & & \\
\hline \multicolumn{15}{|l|}{ F12 (rs1801020) } \\
\hline Cohort & $\mathrm{A} 1$ & $\mathrm{~A} 2$ & N & BETA & SE & $P$ & MAF & HetISq & HetP & A/A Freq & A/G Freq & G/G Freq & HWE chi-square & HWE-p \\
\hline TSP & A & G & 381 & -0.061 & 0.031 & 0.0459 & 0.23 & & & $4.58 \%$ & $33.75 \%$ & $61.67 \%$ & 0.514 & 0.426 \\
\hline MRS & A & G & 798 & -0.055 & 0.018 & 0.0026 & 0.24 & & & $6.14 \%$ & $35.71 \%$ & $58.15 \%$ & 0.339 & 0.0561 \\
\hline Meta-analysis & A & G & 1179 & -0.057 & 0.016 & 0.0003 & 0.24 & 0 & 0.947 & & & & & \\
\hline
\end{tabular}

A1/A2: effect allele/non-effect allele, N: sample size, BETA: estimated beta coefficient, SE: standard error of beta, P: $p$-value for beta, MAF: minor allele frequency, HetP: $p$-value for Cochran's Q statistic, HetISq: $I^{2}$ heterogeneity index, TSP: twin \& sibling participants, MRS: Marine resiliency study 


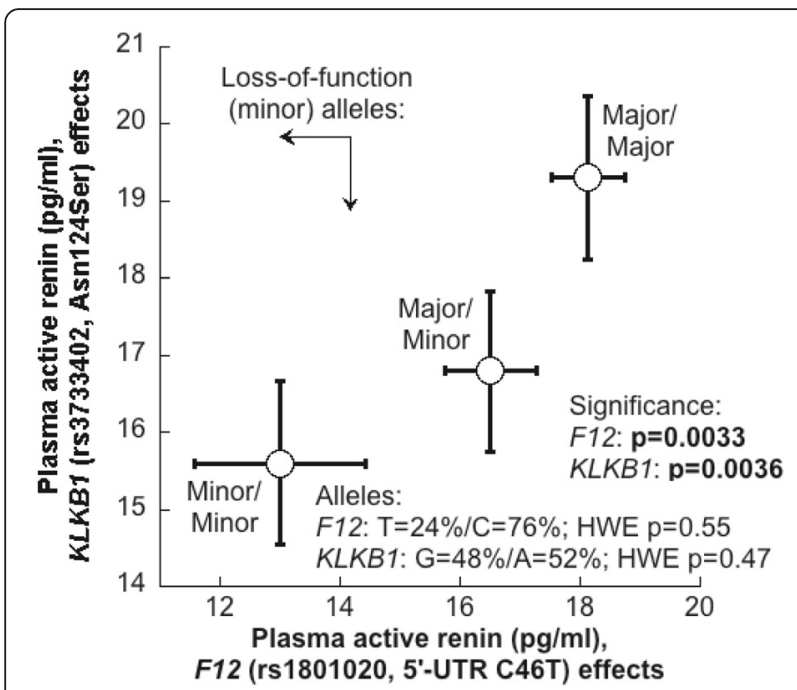

Fig. 1 Effect of polymorphisms in the KLKB1 (Asn124Ser) and F12 (5'-UTR C46T) loci on circulating levels of active renin in UCSD twins/siblings and in MRS subjects. Polymorphisms at F12 and KLKB1 loci influenced plasma active renin levels in two independent cohorts civilian and marines. Each SNP was in Hardy-Weinberg equilibrium with $p>0.05$

Digestion of human recombinant pro-renin with kallikrein (KAL) yields active renin and the pro-peptide byproduct MALDI-TOF analysis of KAL digested pro-renin displayed two peaks of $\mathrm{m} / \mathrm{z} 36,861$ and 5100, corresponding to the theoretical masses of active renin and pro-peptide respectively (Fig. 2, lower panels). In control reaction, where pro-renin was incubated in absence of KAL, MS chromatogram showed a single peak of $\mathrm{m} / \mathrm{z} 44,255$, representing the intact pro-renin (Fig. 2, upper panel). In order to identify the sequence of the digested products, the digestion mixture was subjected to SDS-PAGE on a $10 \%$ gel to separate high molecular weight pro-renin and active renin, and on a 4-12\% gradient gel to separate low molecular weight pro-peptide fragment. A faster migrating band compared to that of pro-renin appeared only in the KAL digested sample (Fig. 3a, marked with arrow 2). Generation of a low molecular weight fragment of $\sim 5 \mathrm{kDa}$ was evidenced after digestion of pro-renin with KAL (Fig. 3a, right panel, marked with arrow 3). Fragments marked with arrow 2 and 3 were cut out from the gel, trypsin digested and subjected to LC-MS analysis for identification. Peptides identified from gel fragment 3 showed significant coverage on the $\mathrm{N}$ and C-terminal of pro-peptide sequence (Fig. 3b), whereas same from gel fragment 2 showed coverage on active-renin (Fig. 3b). Since LC-MS analysis from gel fragment 3 identified some active renin sequence and gel fragment 2 identified some pro-peptide sequence, we quantified the data by normalizing the total sum of spectra for pro-peptide and active renin observed in gel fragment 2 and 3 by their amino acid length. Quantification of mass spec data showed a significant enrichment (400-fold) of pro-peptide to active renin ratio in gel fragment 3 over gel fragment 2 (Fig. 3c).

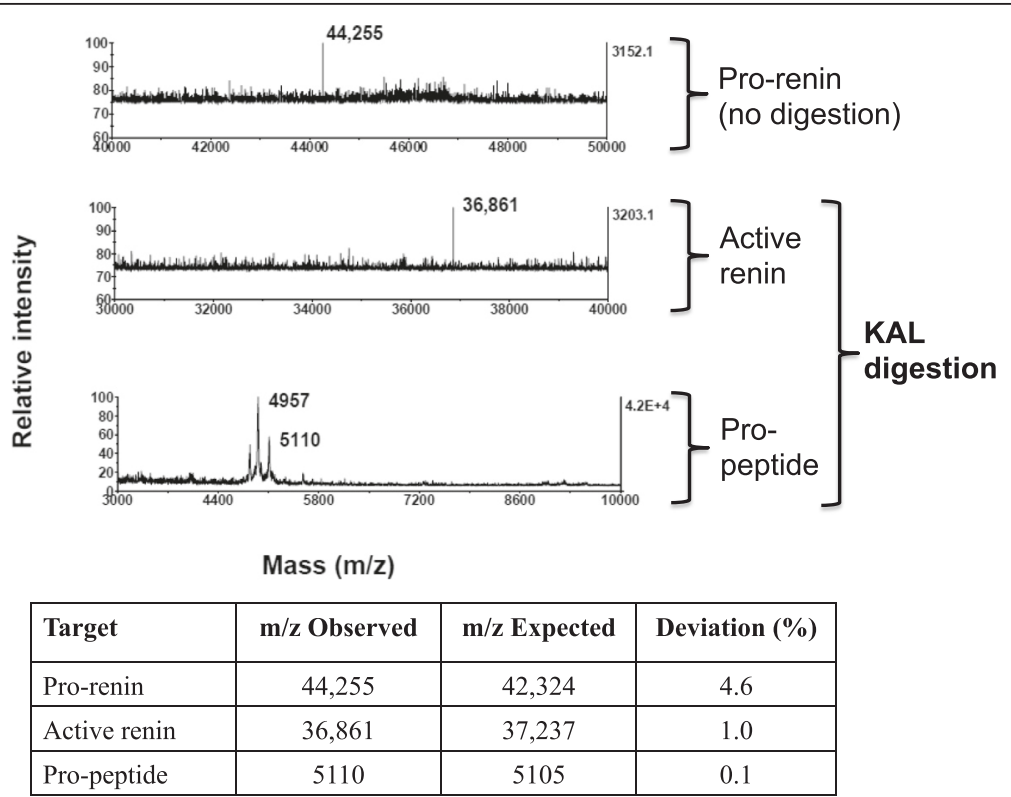

Fig. 2 Mass spectrometric analysis of the KAL digested samples of recombinant pro-renin. Recombinant pro-renin was incubated in absence (upper panel) or presence (middle and bottom panel) of KAL in the assay buffer as mentioned before. The digestion mixture was acidified and purified through ZipTip and subjected to MALDI-TOF analysis in linear mode. Observed masses were compared with the theoretical mass predicted by ProteinProspector program and are shown in the table 


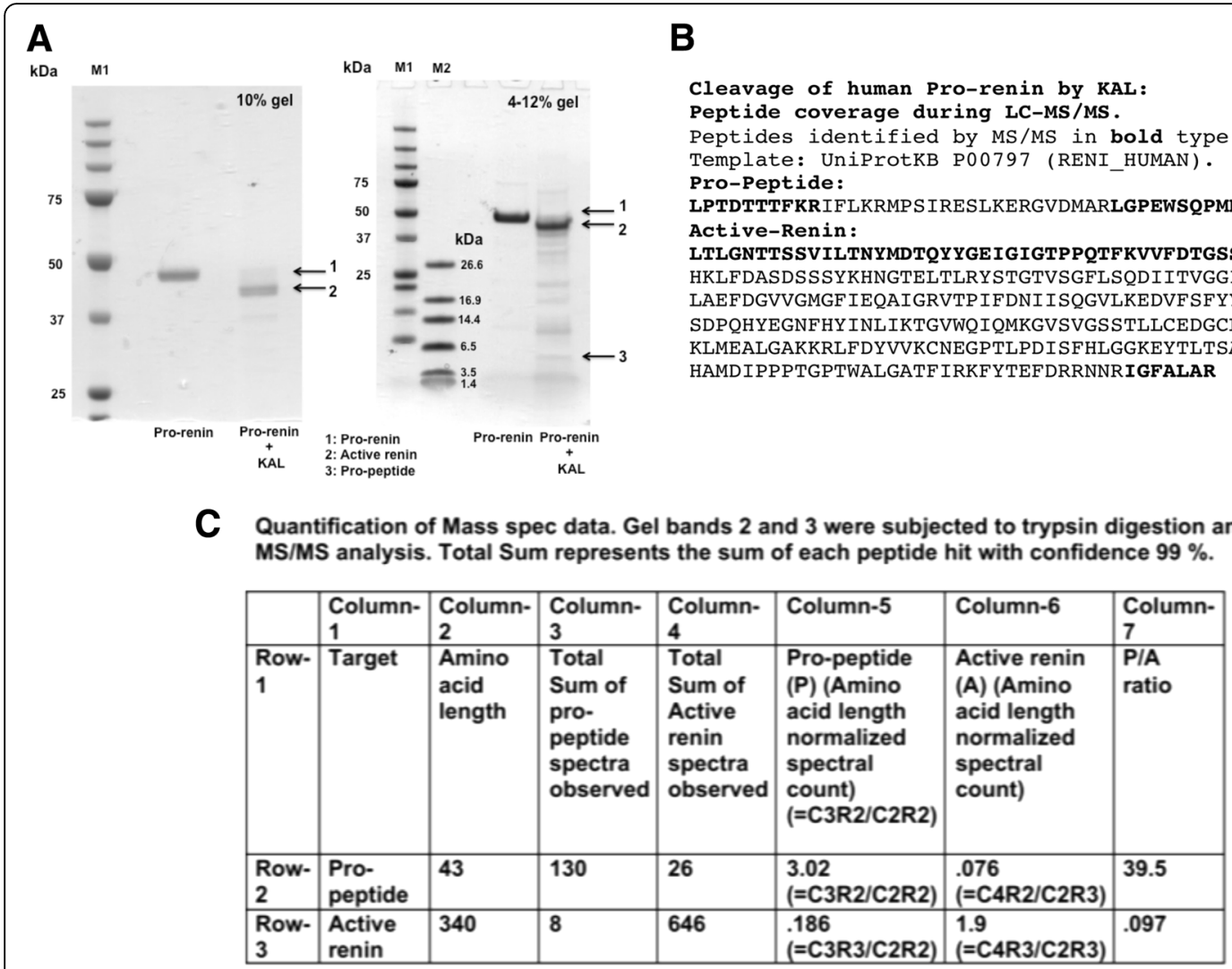

Fig. 3 Molecular mass analysis of fragments generated by KAL digestion of pro-renin; identification of active renin and pro-peptide fragments. a Recombinant pro-renin was digested with KAL as described in Fig. 2 and subjected to SDS-PAGE; $10 \%$ gel (left panel) for separation of active renin from pro-renin and 4-12\% gel (right panel) for identification of lower ( 5 kDa) fragment). Fragments were numbered as 1 (pro-renin), 2 (active renin) and 3 (pro-peptide). M1 and M2 are SDS-PAGE molecular weight standards in broad range and low range respectively $\mathbf{b}$ Fragment 2 and 3 were cut out and subjected to trypsin digestion and LC-MS/MS analysis separately for identification. Peptide coverage (bold type) identified by MS/MS in the termini of pro-peptide (from fragment 3) and active renin (from fragment 2) is shown. c Quantification of the MS data. The total sum of pro-peptide and active renin spectra observed were normalized by the amino acid length (43 for pro-peptide and 340 for the active renin) to represent the pro-peptide to active renin ratio in gel fragments 2 or 3

KAL digested pro-renin cleaves angiotensinogen substrate to generate angiotensin I

Active renin digests substrate angiotensinogen to generate angiotensin I (Ang I). We tested the ability of KAL digested pro-renin to digest angiotensinogen. The pre-angiotensinogen 1-14 tetra deca peptide (AGT) was incubated with the KAL-digested prorenin. Analysis of the digestion reaction containing AGT and KAL revealed one major peptide of $\mathrm{m} / \mathrm{z}$ 1759.9 (Fig. 4a, upper panel). Incubation of pro-renin with KAL followed by the addition of AGT generated a major peak of $\mathrm{m} / \mathrm{z} 1296.81$ (Fig. 4a, lower panel). MS/MS analysis of the precursor mass 1759.9 and 1296.8 confirmed the sequence of these two peptides as amino acids 34-47 and 34-43 of human angiotensinogen (Fig. 4b). Quantification of MS data suggest $96 \%$ generation of Ang I peptide in reaction containing KAL, pro-renin and AGT, whereas only $24 \%$ in presence of pro-renin and AGT and $8 \%$ in presence of KAL and AGT. The generation of Ang I or AGT 1-10 peptide of $\mathrm{m} / \mathrm{z} 1296.8$ was not detected in digestion reactions containing only KAL, pro-renin, $\mathrm{AGT}$ or in KAL and prorenin combination (Fig. 4c).

\section{Renin co-localized with KAL, in kidney JG cells and their renin secretory granules}

Immunofluorescence experiments of mouse juxtaglomerular cells (Fig. 5a) as well as in mouse kidney section (Fig. 5b), was used to establish renin's subcellular co-localization with its processing enzyme KAL. The immunofluorescence micrographs showed that renin and KAL co-localized partially as evidenced by the orange/yellow fluorescence in the overlay figures. Pearson coefficient of co-localization was 0.15 for As1.4 cells and 0.5 for the kidney section.

\section{Endogenous expression of KLKB1 and REN}

After confirming by in vitro assay that KAL processed prorenin to active renin, we analyzed how the expressions of KLKB1 and REN genes might be correlated 


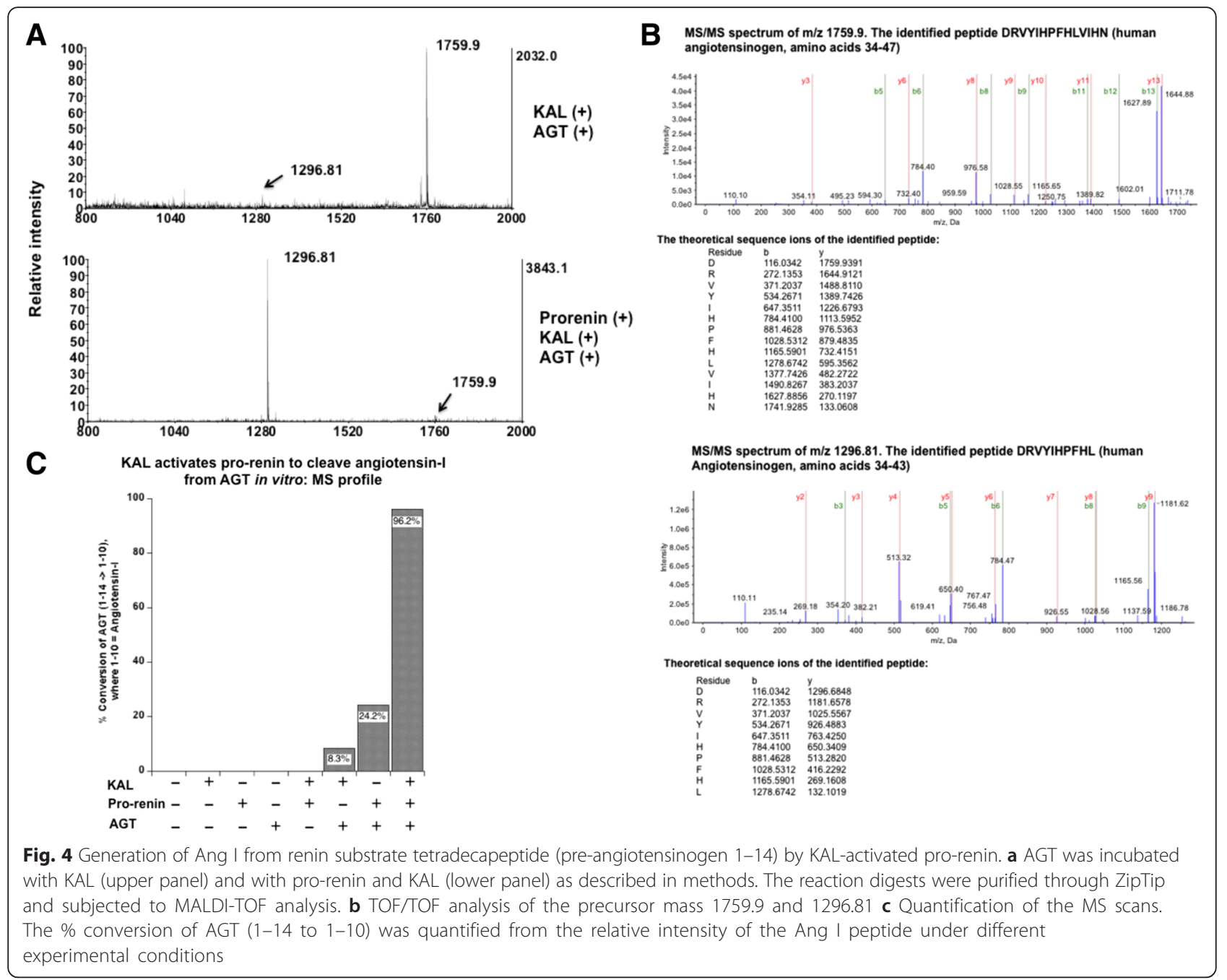

under various physiological conditions. REN and KLKB1 mRNA expression data were collected and analyzed for in mouse As4.1 cells (Fig. 6a) and adrenal tissues of rodent genetic hypertension models: blood pressure low (BPL) and blood pressure high (BPH) mouse models and normotensive Wistar-Kyoto (WKY) and spontaneously hypertensive (SHR) rat models (Fig. 6b). In As4.1 cells feedback inhibition of renin expression was observed by the addition of interleukin $1-\beta$ or hydrogen peroxide, concomitantly KLKB1 expression remained unaltered. The hypotensive phenotype of BPL mice triggered renin expression, $\sim 4$ fold higher compared to hypertensive BPH mice. However the expression of KLKB1 did not differ significantly amongst BPL and $\mathrm{BPH}$ mice. The normotensive WKY rats have significantly higher KLKB1 expression ( 5.5 fold) compared to the hypertensive SHR rats with more or less similar level of REN mRNA expression in both rat models. Thus regulation of blood pressure under various physiological conditions may involve modulation in the expression of either REN or KLKB1.

\section{Discussion}

In vitro studies have demonstrated that proteases such as trypsin, plasmin, pepsin, kallikrein and several others activate zymogen pro-renin to active renin [35-38]. Studies before the era of mass spectroscopy suggested involvement of KLKB1 and FXIIa in pro-renin processing [39-41]. Genetic variation at the KLKB1 locus (encoding for plasma pre-kallikrein or Fletcher factor; EC 3.4.21.34) was previously most widely investigated for its roles in coagulation and allergy. We demonstrate using in vitro enzymatic assay the ability of active protease KAL in processing pro-renin $\rightarrow$ renin. A second association of renin activity and the protease F12 locus (encoding for Factor XII or Hageman factor; EC 3.4.21.38) suggests a cascade of enzymatic events 


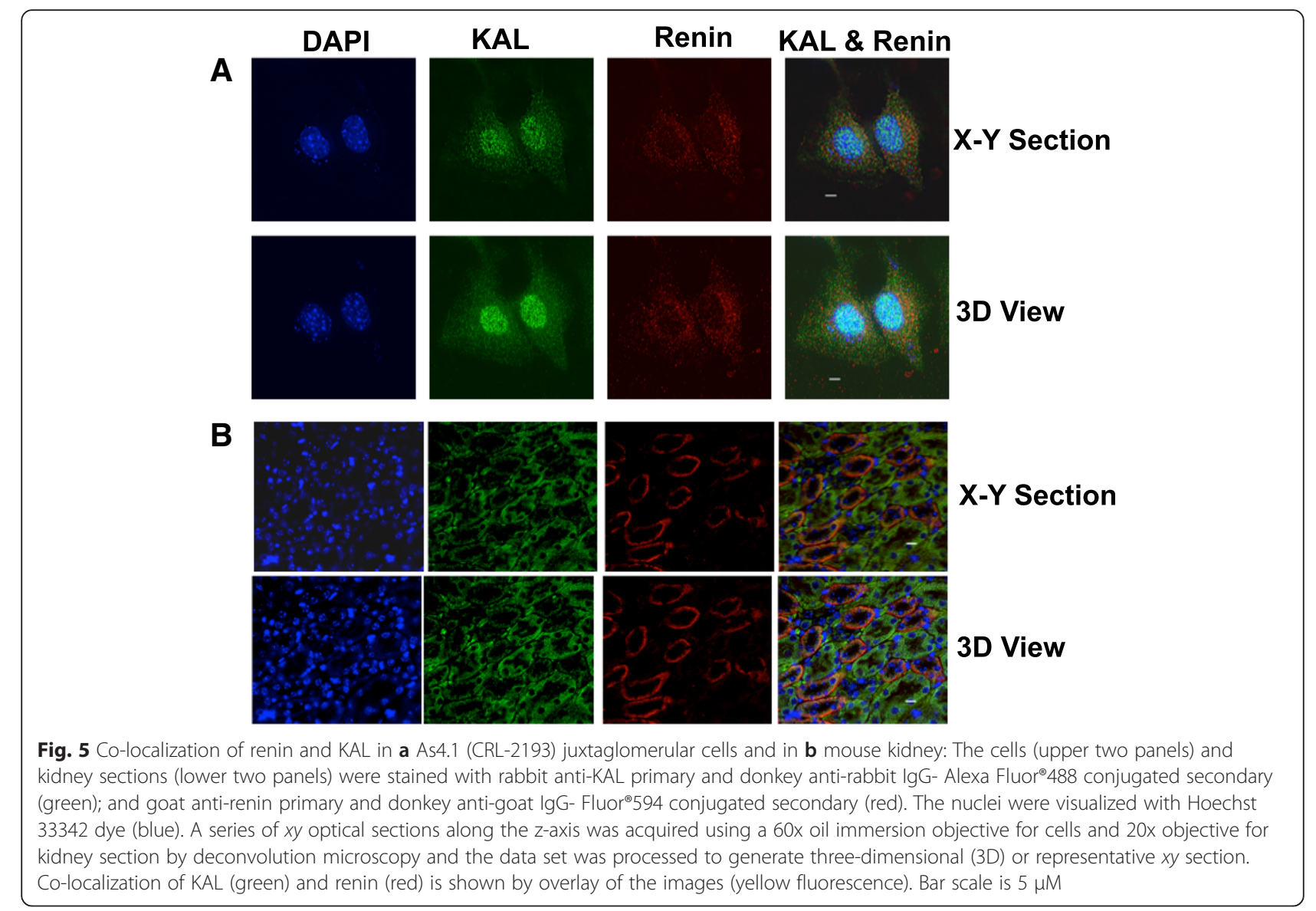

$($ FXIIa $\rightarrow$ KAL) in control of pro-renin activation. Generation of active renin by the cascade thus provides evidence of a site for BP regulation.

The KLKB1 locus lies directly beneath a previously described LOD peak $(\mathrm{LOD}=3.2)$ for $\mathrm{BP}$ on chromosome 8 in the genetically hypertensive strain of mice (BPH) [42]. We therefore explored the effects of $K L K B 1$ genetic variation upon formation of active renin. While most of the KLKB1 single nucleotide polymorphisms (SNPs) reported are located in the non-coding regions, rs3733402
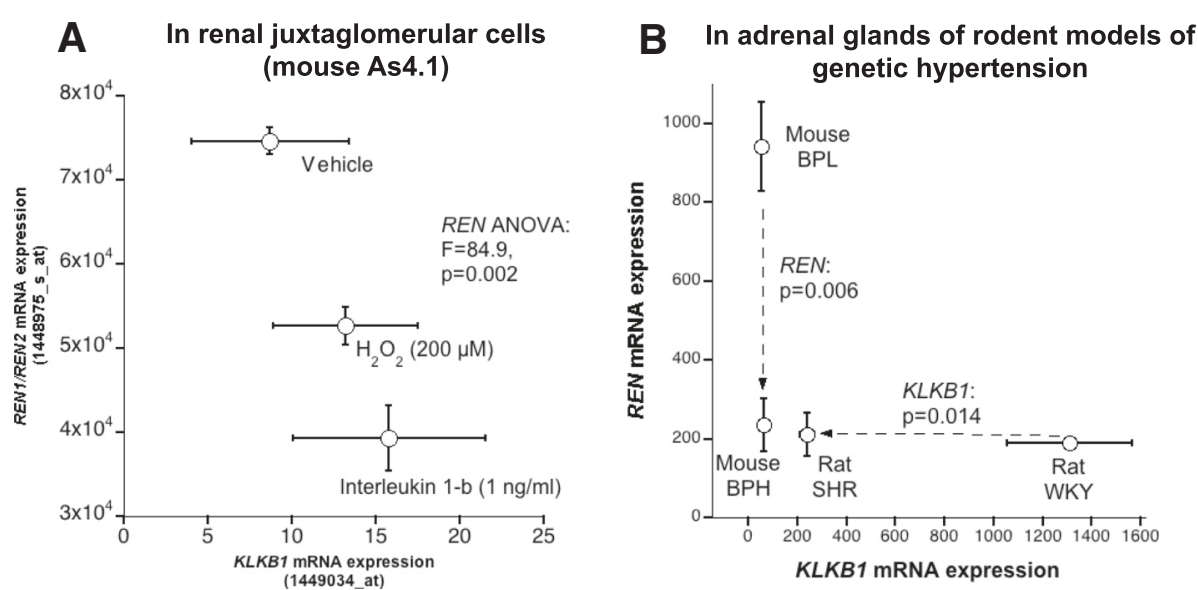

Fig. 6 Correlation of REN and KLKB1 mRNA expression in a renal juxtaglomerular cells and (B) in the adrenal glands of rodent models of genetic hypertension (mouse: BPH/BPL and rat: SHRMKY): REN and KLKB1 mRNA expression data were collected and compared for (a and b) 
in exon 5 results in an amino acid substitution Asn124Ser [14, 33]. This mutation in the apple domain 2 of heavy chain reduces the binding of KAL to its substrate HMWK, and therefore this SNP was chosen to investigate its association with prorenin processing. Indeed, an immunoassay specific for active renin revealed that Ser/Ser homozygotes had lower circulating active renin (Fig. 1), consistent with diminished pro-renin cleavage by a less active Ser allele. Previously, rs3733402 has shown strong association with pre-pro-endothelin-1 and pre pro-adrenomedullin in the Prevention of Renal and Vascular End stage disease (PREVEND) study [43]. In the recent study by Lieb et al. the top SNPs identified were rs12374220, an intronic variant in the TENM3 gene, rs5030062 in the intron 6 of kininogen 1 gene and rs4253311 in intron 11 of the kallikrein $\mathrm{B}$ (KLKB1) gene. The intronic SNP rs4253311 provided no evidence for association with renin concentrations and explained $0.87 \%$ of plasma renin activity variance [44]. In our study MALDI mass spectrometry documented the formation of active renin and the pro-peptide after digestion of pro-renin with KAL (Fig. 2 \& Fig. 3). Furthermore the sub-cellular co-localization of renin with KAL suggests molecular interaction between these two proteins (Fig. 5a \&b). Renin immunoreactivity has previously been shown in the cytoplasmic granules of cultured JG cells and in kidney sections [45]. The cleavage sites involved in pro-renin processing include lysine-arginine, which is the recognition site of plasma kallikrein [46]. Our genetic and biochemical data suggests an enzyme-substrate relation between KAL and prorenin. This suggests the possible existence of feedback regulation at the molecular level in the events leading to active renin generation by KAL and $\mathrm{BP}$ regulation.

KAL is a glycoprotein that takes part in the surface dependent activation of blood coagulation, fibrinolysis and kinin generation. It is synthesized in the liver and secreted into the blood as prekallikrein, which is then converted to active plasma kallikrein by factor FXIIa [47]. The C46T 5'-UTR polymorphism associated with Hageman factor has been described to be associated with its plasma concentration and thrombotic risk $[48,49]$. The KAL protease might catalyzes the conversion of HMWK to bradykinin in one hand, and the active renin on other hand. The downstream target angiotensin converting enzyme (ACE) then modulates the concentration of angiotensin II, the key player of the RAAS system, and bradykinin, a component of the kallikrein-kinin system in opposite direction, therefore establishing a direct interaction between kallikrein-kinin and renin-angiotensin system [50, 51].

The genetic variation in the F12 and KLKB1 loci directly affecting their amino acid sequence could ultimately influenced the processing, secretion or circulation of the active renin protein, which in turn mediates the BP phenotype. Allelic effects might also act on the cluster of characteristics associated with cardiovascular risk for which plasma renin is a biomarker. In the coagulation system, it has been reported that even the homozygous deficiency of the $K L K B 1$ loci results in no discernible coagulopathy [52]. In treatment of hereditary angioedema inhibition of KAL does play a beneficial role, perhaps by inhibition of bradykinin formation [53].

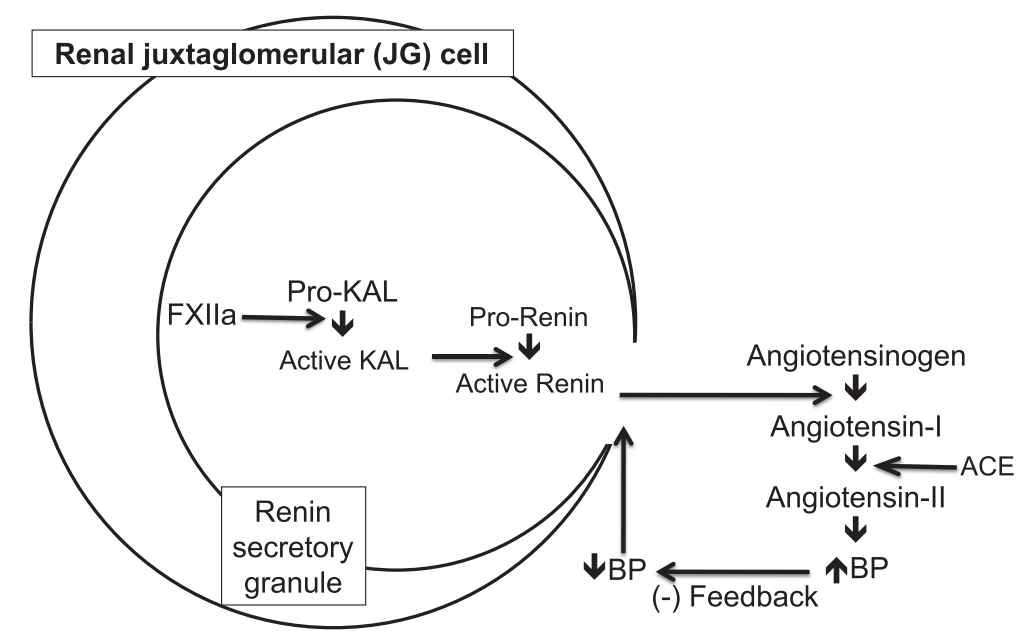

Fig. 7 Hypothetical schematic representing activation of pro-renin by a proteolytic enzyme cascade of FXIla $\rightarrow$ KAL, with consequences for regulation of BP. Pro-renin processing within the secretory granule of renal juxtaglomerular cells by sequential enzymatic events catalyzed by $\mathrm{F} 12$ and KAL 


\section{Advantages and limitations}

Here we report a comprehensive GWAS showing correlation between polymorphisms at two independent loci (KLKB1 rs3733402 and F12 rs1801020) and plasma renin activity. Cellular and biochemical evidence is provided to establish that correlation. To our knowledge this is the first report of SNPs in two independent loci with significant trait association with activation of renin-angiotensin system. This study focused on the best characterized SNP (rs3733402) in the exon 5 of KLKB1 gene. Although association of kallikrein with renin activation has previously been described, adequate information on direct in vitro protease biochemistry was lacking. Therefore we used a mass spectrometry approach to characterize in vitro digestion of prorenin by KAL to reestablish kallikrein association with prorenin processing. In the scenario of this genetic association, the efficacy of digestion of prorenin by mutant KAL (Asp124Ser) needs to be compared with that of the wild type KAL. We have not addressed in these populations the active plasma renin association with the previously described intronic variant at KLKB1 (rs4253311) and other SNPs. Future studies will explore the association of these two SNPs with BP, renal and/or metabolic traits.

\section{Conclusion}

Our findings draw attention to the role of KAL as a pro-renin convertase and suggest a potential target for inhibition of the rate-limiting step in the RAS pathway. Polymorphisms at the KLKB1 (rs3733402) and F12 (rs1801020) loci are associated with low active plasma renin activity. Genetic, cell and biochemical studies suggest a cascade of enzymatic events involving factor FXIIa activation of prekallikrein to active kallikrein in control of pro-renin activation. Thus plasma kallikrein presents potential as novel therapeutic target for blood pressure regulation with implications of KAL inhibition for treatment of hypertension (Fig. 7).

\section{Abbreviations}

AGT: Angiotensinogen; Ang I: Angiotensin I; Ang II: Angiotensin II; BP: Blood pressure; Factor XII (FXII Hageman factor): protein encoded by gene F12; JG: Juxtaglomerular; KAL: Kallikrein; MAF: Minor allele frequency; MALDI-TOF: Matrix Assisted Laser Desorption Ionization/Time Of Flight: Prekallikrein (prokallikrein Fletcher factor): protein encoded by gene KLKB1; RAAS: Renin-angiotensin-aldosterone system; SNPs: Single nucleotide polymorphisms; TSP: Twin and sibling participants.

\section{Competing interests}

The authors declare that they have no competing interests.

\section{Authors' contributions}

NB carried out the biochemical and cell biology studies \& wrote the manuscript; AXM, FR,KZ did the genetic and statistical analysis; SAM did immunofluorescence and manuscript preparation; SK,MM,KZ \&CMH did the biochemical assays; RSF did the transcriptome studies; SKM helped in the experimental studies. DGB and CMN did the human studies and helped write the manuscript. SV helped in study design, experimentation and writing of the manuscript; DTOC conceived of the study, its design and coordination and helped to draft the manuscript.

\section{Support}

Support for these studies came from National Institutes of Health Grants R01-DK094894 (to D.T.O.C. and S.M.V.), R01MH093500 (to C.M.N), R01HL108629 (to S.M.V). Funding for data collection from the MRS cohort came from Veterans Affairs Health Service Research and Development project SDR 09-0128, the Marine Corps, and the Navy Bureau of Medicine and Surgery (to D.G.B).

\section{Author details}

${ }^{1}$ Departments of Medicine, University of California at San Diego, 9500 Gilman Drive, La Jolla 92093-0838, USA. ² Veteran Affairs (VA) San Diego Health Care System and VA Center of Excellence for Stress and Mental Health, $3350 \mathrm{La}$ Jolla Village Drive, San Diego 92161, USA. ${ }^{3}$ Department of Psychiatry, University of California at San Diego, 9500 Gilman Drive, La Jolla 92093-0838, USA. ${ }^{4}$ Departments of Pharmacology, Institute of Genomic Medicine,

University of California at San Diego, 9500 Gilman Drive, La Jolla 92093-0838, USA.

Received: 5 August 2015 Accepted: 1 March 2016

Published online: 11 March 2016

\section{References}

1. Ezzati M, Lopez AD, Rodgers A, Vander Hoorn S, Murray CJ. Selected major risk factors and global and regional burden of disease. Lancet. 2002;360: $1347-60$

2. Navar LG. Intrarenal renin-angiotensin system in regulation of glomerular function. Curr Opin Nephrol Hypertens. 2014;23:38-45.

3. Sarafidis PA, Ruilope LM. Aggressive blood pressure reduction and renin-angiotensin system blockade in chronic kidney disease: time for re-evaluation? Kidney Int. 2014;85(3):536-46

4. Morris BJ. Renin, Genes, MicroRNAs, and Renal Mechanisms Involved in Hypertension. Hypertension. 2015;65:956-62.

5. Zhuo JL, Ferrao FM, Zheng Y, Li XC. New frontiers in the intrarenal Renin-Angiotensin system: a critical review of classical and new paradigms. Front Endocrinol. 2013;4:166.

6. Carey RM, Siragy HM. Newly recognized components of the renin-angiotensin system: potential roles in cardiovascular and renal regulation. Endocr Rev. 2003:24(3):261-71.

7. Crowley SD, Gurley SB, Herrera MJ, Ruiz P, Griffiths R, Kumar AP, et al. Angiotensin II causes hypertension and cardiac hypertrophy through its receptors in the kidney. Proc Natl Acad Sci U S A. 2006;103(47):17985-90.

8. Reudelhuber TL. Reudelhuber interaction between prorenin, renin and the (pro) renin receptor: time to rethink the role in hypertension. Curr Opin Nephrol Hypertens. 2012;21:137-41. SRC - GoogleScholar.

9. Nylocks KM, Michopoulos V, Rothbaum AO, Almli L, Gillespie CF, Wingo A, et al. An angiotensin-converting enzyme (ACE) polymorphism may mitigate the effects of angiotensin-pathway medications on posttraumatic stress symptoms. Am J Med Genet B Neuropsychiatr Genet. 2015;168B:307-15.

10. Brudey C, Park J, Wiaderkiewicz J, Kobayashi I, Mellman TA, Marvar PJ. Autonomic and Inflammatory Consequences of Posttraumatic stress disorder (PTSD) and the Link to Cardiovascular Disease. Am J Physiol Regul Integr Comp Physiol. 2015.

11. Hsueh WA, Baxter JD. Human prorenin. Hypertension. 1991:17(4):469-77.

12. Castrop H, Höcherl K, Kurtz A, Schweda F, Todorov V, Wagner C. Physiology of kidney renin. Physiol Rev. 2010:90(2):607-73.

13. Hall JE. Thekidney, hypertension, and obesity. Hypertension. 2003;41:625-33.

14. Katsuda I, Maruyama F, Ezaki K, Sawamura T, Ichihara Y. A new type of plasma prekallikrein deficiency associated with homozygosity for Gly104Arg and Asn124Ser in apple domain 2 of the heavy-chain region. Eur J Haematol. 2007;79(1):59-68.

15. Cockburn MG, Hamilton A, Zadnick J, Cozen W, Mack TM. The occurrence of chronic disease and other conditions in a large population-based cohort of native Californian twins. Twin Res. 2002;5(5):460-7.

16. Zhang L, Rao F, Wessel J, Kennedy BP, Rana BK, Taupenot L, et al. Functional allelic heterogeneity and pleiotropy of a repeat polymorphism in tyrosine hydroxylase: prediction of catecholamines and response to stress in twins. Physiol Genomics. 2004;19(3):277-91. 
17. Deinum J, Derkx FH, Schalekamp MA. Improved immunoradiometric assay for plasma renin. Clin Chem. 1999;45(6 Pt 1):847-54.

18. Johnson AD, Handsaker RE, Pulit SL, Nizzari MM, O'Donnell CJ, de Bakker PIW. SNAP: a web-based tool for identification and annotation of proxy SNPs using HapMap. Bioinformatics (Oxford, England). 2008;24(24):2938-9.

19. Baker DG, Nash WP, Litz BT, Geyer MA, Risbrough VB, Nievergelt CM, et al. Predictors of Risk and Resilience for Posttraumatic Stress Disorder Among Ground Combat Marines: Methods of the Marine Resiliency Study. Prev Chronic Dis. 2012;9:110134

20. Nievergelt CM, Maihofer AX, Mustapic M, Yurgil KA, Schork NJ, Miller MW. Genomic predictors of combat stress vulnerability and resilience in US Marines a genomewide association study across multiple ancestries implicates PRTFDC1 as a potential PTSD gene. Psychoneuroendocrinology. 2014;51:459-71

21. Nievergelt CM, Wineinger NE, Libiger O, Pham P, Zhang G, Baker DG, et al. Chip-based direct genotyping of coding variants in genome wide association studies: utility, issues and prospects. Gene. 2014;540(1):104-9.

22. Purcell S, Neale B, Todd-Brown K, Thomas L, Ferreira MAR, Bender D, et al. PLINK: a tool set for whole-genome association and population-based linkage analyses. Am J Hum Genet. 2007;81(3):559-75.

23. Nievergelt CM, Maihofer AX, Shekhtman T, Libiger O, Wang X, Kidd KK, et al. Inference of human continental origin and admixture proportions using a highly discriminative ancestry informative 41-SNP panel. Investigative Genet. 2013:4(1):13

24. Purcell S, Cherny SS, Sham PC. Genetic Power Calculator: design of linkage and association genetic mapping studies of complex traits. Bioinformatics. 2003;1:149-50

25. Willer CJ, Li Y, Abecasis GR. METAL: fast and efficient meta-analysis of genomewide association scans. Bioinformatics (Oxford, England). 2010; 26(17):2190-219.

26. Biswas N, Rodriguez-Flores JL, Courel M, Gayen JR, Vaingankar SM, Mahata $M$, et al. Cathepsin L colocalizes with chromogranin a in chromaffin vesicles to generate active peptides. Endocrinology. 2009;150(8):3547-57.

27. Biswas N, Friese RS, Gayen JR, Bandyopadhyay G, Mahata SK, O'Connor DT. Discovery of a novel target for the dysglycemic chromogranin A fragment pancreastatin: interaction with the chaperone GRP78 to influence metabolism. PloS ONE. 2014;9:e84132.

28. Courel M, Vasquez MS, Hook VY, Mahata SK, Taupenot L. Sorting of the neuroendocrine secretory protein Secretogranin II into the regulated secretory pathway: role of $\mathrm{N}$ - and C-terminal alpha-helical domains. J Biol Chem. 2008;283(17):11807-22.

29. Ott HC, Matthiesen TS, Goh SK, Black LD, Kren SM, Netoff TI, et al. Perfusion-decellularized matrix: using nature's platform to engineer a bioartificial heart. Nat Med. 2008;14(2):213-21.

30. Friese RS, Ye C, Nievergelt CM, Schork AJ, Mahapatra NR, Rao F, et al Integrated computational and experimental analysis of the neuroendocrine transcriptome in genetic hypertension identifies novel control points for the cardiometabolic syndrome. Circ Cardiovasc Genet. 2012;5(4):430-40.

31. Friese RS, Schmid-Schönbein GW, O'Connor DT. Systematic polymorphism discovery after genome-wide identification of potential susceptibility loci in a hereditary rodent model of human hypertension. Blood Press. 2011;20(4):222-31.

32. Thompson HA, Burson JM, Lang JA, Gross KW, Sigmund CD. Tissuespecific expression of novel messenger ribonucleic acids cloned from a renin-expressing kidney tumor cell line (As4.1). Endocrinology. 1995; 136(7):3037-45

33. Yu H, Anderson PJ, Freedman BI, Rich SS, Bowden DW. Genomic structure of the human plasma prekallikrein gene, identification of allelic variants, and analysis in end-stage renal disease. Genomics. 2000;69(2):225-34.

34. Calafell F, Almasy L, Sabater-Lleal M, Buil A, Mordillo C, Ramírez-Soriano A, et al. Sequence variation and genetic evolution at the human F12 locus: mapping quantitative trait nucleotides that influence FXII plasma levels. Hum Mol Genet. 2010;19(3):517-25.

35. Takahashi S, Murakami K, Miyake Y. Activation of kidney prorenin by kidney cathepsin B isozymes. J Biochem. 1982;91(1):419-22.

36. Wu Z, Cappiello MG, Scott BB, Bukhtiyarov Y, McGeehan GM. Purification and characterization of recombinant human renin for $X$-ray crystallization studies. BMC Biochem. 2008;9:19.

37. Reudelhuber TL, Ramla D, Chiu L, Mercure C, Seidah NG. Proteolytic processing of human prorenin in renal and non-renal tissues. Kidney Int. 1994:46(6):1522-4
38. Morris BJ, McGirr JG. Direct evidence, using Pro-Phe-ArgCH2Cl, that plasama kallikrein has a role in acid activation of inactive renin in plasma from normal subjects. Biomed Res. 1981;2(5):552-9.

39. Sealey JE, Atlas SA, Laragh JH, Silverberg M, Kaplan AP. Initiation of plasma prorenin activation by Hageman factor-dependent conversion of plasma prekallikrein to kallikrein. Proc Natl Acad Sci U S A. 1979;76(11):5914-8.

40. Rumpf KW, Becker K, Kreusch U, Schmidt S, Vetter R, Scheler F. Evidence for a role of plasma kallikrein in the activation of prorenin. Nature. 1980; 283(5746):482-3.

41. Derkx FH, Schalekamp MP, Bouma B, Kluft C, Schalekamp MA. Plasma kallikreinmediated activation of the renin-angiotensin system does not require prior acidification of prorenin. J Clin Endocrinol Metab. 1982;54(2):343-8.

42. Wright FA, O'Connor DT, Roberts E, Kutey G, Berry CC, Yoneda LU, et al. Genome scan for blood pressure loci in mice. Hypertension. 1999;34(4 Pt 1): 625-30.

43. Verweij N, Mahmud $H$, Mateo Leach I, de Boer RA, Brouwers FP, Yu H, et al. Genome-wide association study on plasma levels of midregionalproadrenomedullin and C-terminal-pro-endothelin-1. Hypertension. 2013; 61(3):602-8.

44. Lieb W, Chen M-H, Teumer A, de Boer RA, Lin H, Fox ER, et al. Genomewide meta-analyses of plasma renin activity and concentration reveal association with the kininogen 1 and prekallikrein genes. Circ Cardiovasc Genet. 2015;8(1):131-40.

45. Hunt MK, Ramos SP, Geary KM, Norling LL, Peach MJ, Gomez RA, et al. Colocalization and release of angiotensin and renin in renal cortical cells. Am J Physiol. 1992:263(3 Pt 2):F363-73.

46. Metters KM, Rossier J, Paquin J, Chrétien M, Seidah NG. Selective cleavage of proenkephalin-derived peptides (less than 23,300 daltons) by plasma kallikrein. J Biol Chem. 1988;263(25):12543-53.

47. Bjorkqvist J, Jamsa A, Renne T. Plasma kallikrein: the bradykinin-producing enzyme. Thromb Haemost. 2013;110:399-407.

48. Bach J, Endler G, Winkelmann BR, Boehm BO, Maerz W, Mannhalter C, et al. Coagulation factor XII (FXII) activity, activated FXII, distribution of FXII C46T gene polymorphism and coronary risk. JTH. 2008;6(2):291-6.

49. Soria JM, Almasy L, Souto JC, Bacq D, Buil A, Faure A, et al. A quantitativetrait locus in the human factor XII gene influences both plasma factor XII levels and susceptibility to thrombotic disease. Am J Hum Genet. 2002; 70(3):567-74

50. Schmaier $\mathrm{AH}$. The kallikrein-kinin and the renin-angiotensin systems have a multilayered interaction. Am J Physiol Regul Integr Comp Physiol. 2003;285: R1-R13.

51. Shen B, El-Dahr SS. Cross-talk of the renin-angiotensin and kallikrein-kinin systems. Biol Chem. 2006;387(2):145-50.

52. Giangrande PL. Six characters in search of an author: the history of the nomenclature of coagulation factors. Br J Haematol. 2003;121:703-12.

53. Cicardi M, Levy RJ, McNeil DL, Li HH, Sheffer AL, Campion M, et al. Ecallantide for the treatment of acute attacks in hereditary angioedema. N Engl J Med. 2010;363(6):523-31.

\section{Submit your next manuscript to BioMed Central and we will help you at every step:}

- We accept pre-submission inquiries

- Our selector tool helps you to find the most relevant journal

- We provide round the clock customer support

- Convenient online submission

- Thorough peer review

- Inclusion in PubMed and all major indexing services

- Maximum visibility for your research

Submit your manuscript at www.biomedcentral.com/submit 